\title{
Beyond Matroids: Secretary Problem and Prophet Inequality with General Constraints
}

\author{
Aviad Rubinstein ${ }^{*}$ \\ UC Berkeley, USA \\ aviad@eecs.berkeley.edu
}

\begin{abstract}
We study generalizations of the "Prophet Inequality" and "Secretary Problem", where the algorithm is restricted to an arbitrary downward-closed set system. For $\{0,1\}$ values, we give $O(\log n)$-competitive algorithms for both problems. This is close to the $\Omega(\log n / \log \log n)$ lower bound due to Babaioff, Immorlica, and Kleinberg. For general values, our results translate to $O(\log n \cdot \log r)$-competitive algorithms, where $r$ is the cardinality of the largest feasible set. This resolves (up to the $O(\log r \cdot \log \log n)$ factors) an open question posed to us by Bobby Kleinberg [13].
\end{abstract}

\section{Categories and Subject Descriptors}

F.1.2 [Modes of Computation]: [Online computation]

\section{General Terms}

Algorithms, Theory

\section{Keywords}

Prophet Inequality, Secretary Problem

\section{INTRODUCTION}

The "Secretary Problem" and "Prophet Inequality" and their variants have been central problems in optimal stopping theory for decades. In both problems, an online decision maker selects one of $n$ items that arrive online. The decision maker observes only one item at a time, and must decide immediately and irrevocably whether to select this item. The expected value of the item selected by the decision maker is compared to the offline optimum. There is

\footnotetext{
*Most of the research was done while the author was an intern at Microsoft Research New England. Part of this research was also supported by Microsoft Research PhD Fellowship, as well as NSF grant CCF1408635 and by Templeton Foundation grant 3966. This work was done in part at the Simons Institute for the Theory of Computing.

Permission to make digital or hard copies of all or part of this work for personal or classroom use is granted without fee provided that copies are not made or distributed for profit or commercial advantage and that copies bear this notice and the full citation on the first page. Copyrights for components of this work owned by others than ACM must be honored. Abstracting with credit is permitted. To copy otherwise, or republish, to post on servers or to redistribute to lists, requires prior specific permission and/or a fee. Request permissions from Permissions@acm.org.

Copyright is held by the owner/author(s). Publication rights licensed to ACM.

STOC'16, June 19-21, 2016, Cambridge, MA, USA

ACM. 978-1-4503-4132-5/16/06...\$15.00

http://dx.doi.org/10.1145/2897518.2897540
}

little that an online algorithm can do in an arbitrary worst case, so the input is restricted: In the Secretary Problem, the values of items are chosen adversarially, but their arrival order is random. In Prophet Inequality, the order is adversarial, but the values are drawn from known, independent but not identical distributions. Algorithms with optimal competitive ratios (with respect to the offline optimum) of $e$ and 2 were known since the 1960's (e.g. [6]) and 1970's [14], respectively.

In the past decade, variants of both problems received significant attention from theoretical computer scientists thanks to their rich algorithmic structure and applications to online and offline mechanism design (e.g. $[2,10,12])$. In particular, there have been many works on settings where the decision maker is allowed to select any subset of the items subject to a certain family of combinatorial constraints. In the famous "Matroid Secretary Problem" [3], for example, the decision maker is allowed to select any independent set in a given matroid. Obtaining a constant competitive ratio algorithm is a long-standing open problem: the state of the art is $O(\log \log r)$, where $r$ is the rank of the matroid $[15,7]$. Its cousin, the "Matroid Prophet Inequality", has been resolved by Kleinberg and Weinberg who gave a 2-competitive algorithm [12]. Other constraints such as special classes of matroids ([4] and references therein), polymatroids [5], etc. have also been studied in these settings. Even the 2013 survey by Dinitz [4] seems outdated with so much exciting progress in the last couple of years (e.g. $[1,8,11,17])$.

\subsection{Our Results}

In this work we revisit an important missing piece in this puzzle: what happens when there are no guarantees on the family of feasible sets? Babaioff, Immorlica, and Kleinberg [3] gave an $\Omega(\log n / \log \log n)$ lower bound ${ }^{1}$ on the competitive ratio of the Secretary Problem with arbitrary downward-closed constraints, even in the special case where all values are in $\{0,1\}$. (Essentially the same construction gives the same lower bound for the corresponding Prophet Inequality; see also Appendix A.) Beyond this lower bound, the problem remained poorly understood. In particular there was a disturbing lack of upper bounds on the competitive ratio - nothing beyond the trivial $O(n)$ was

\footnotetext{
${ }^{1}$ Stated in terms of the $r$, the maximum size of a feasible set, [3]'s construction gives an $\Omega(r)$-lower bound on the competitive ratio; one can easily obtain a matching $O(r)$-upper bound by applying the algorithms for the classical (single item) variants of both problems.
} 
known. Here, we make significant progress towards closing this gap:

TheOREM 1 (MAIN TheOREM). When the items take values in $\{0,1\}$, the competitive ratios for DowNWARDClosed Prophet and Downward-Closed Secretary are $O(\log n)$.

For general (non-negative) valuations, we can reduce to the $\{0,1\}$-valued case with a $\operatorname{loss}$ of $O(\log r)$, where $r$ denotes the cardinality of the largest feasible set.

Corollary 1. For general item values, the competitive ratios for DownWARD-ClOSED PROPHET and DownWARDClosed Secretary are $O(\log n \cdot \log r)$.

Computational Efficiency. Our algorithm for Downward-Closed SeCretary assumes access to a demand-like oracle. The algorithm for DownWARD-Closed PROPHET makes slightly more complicated queries of the form: "conditioning on the history of realizations and items selected by the algorithm so far, what is the probability that the prophet can still obtain value $\tau$ ?". Our algorithms are efficient assuming access to such oracles. Note that it is unreasonable to expect much more: optimizing over arbitrary families of downward-closed sets is computationally intractable even in the offline case (for example, when the feasible sets are the independent sets of a graph [13]).

Non-Monotone Feasibility Constraints. One can further generalize the problem and consider feasibility constraints which are not even downward-closed. In Section 4 we briefly discuss Non-MOnOtone Prophet and NonMONOTONE SECRETARY and prove near tight lower bounds on the competitive ratios.

\subsection{Techniques}

Both algorithms rely on potential function argument. The key idea for the analysis of Downward-ClOsed PropheT is the use of a dynamic potential function: when the algorithm cannot guarantee adequate progress with respect to the current potential function, it dynamically modifies the potential function. We believe that this simple idea will find applications in other potential function arguments.

We note that our algorithms for DOWNWARD-CLOSED Prophet and Downward-Closed SECRETARY bear little resemblance to each other or to related works on the Matroid Secretary Problem and Matroid Prophet Inequality. In particular, we focus on the case of $\{0,1\}$-valued items, which is easy for matroids. Defying our intuition from matroids again, our algorithm and analysis for DownwARDCLOSED SECRETARY happens to be simpler than that of Downward-Closed Prophet. Nevertheless, we begin with Downward-Closed Prophet in the next section, as we find the techniques more exciting. The algorithm for Downward-Closed Secretary is described in Section 3 and non-monotone constraints are discussed in Section 4.

\section{PROPHET}

Definition 1. (DownWARd-Closed Prophet) Consider $n$ items with independently distributed values $\left\{X_{i} \sim \mathcal{D}_{i}\right\}_{i=1}^{n}$. Let $\mathcal{F}$ be an arbitrary downward-closed set system over the items $[n]$, and initialize $W$ as the empty set. The algorithm receives as input $n, \mathcal{F}$, and the distributions, and observes the realizations (the value of $X_{i}$ ) online. After observing the realization of $X_{i}$, the algorithm must decide (immediately and irrevocably) whether to add $i$ to the set $W$, subject to the constraint that $W$ remains a feasible set in $\mathcal{F}$. The objective is to maximize the sum of values of items in $W$.

ThEOREM 2. When the $X_{i}$ 's take values in $\{0,1\}$, there is a deterministic algorithm for DOWNWARD-CLOSED PROPHET that achieves a competitive ratio of $O(\log n)$.

Let $r$ denote the maximum cardinality of a feasible set $S \in$ $\mathcal{F}$.

COROLlaRY 2. There is a deterministic algorithm for DOWNWARD-CLOSED PROPHET that achieves a competitive ratio of $O(\log n \cdot \log r)$.

Proof of Corollary 2 from Theorem 2. We recover separately the contributions from "tail" events (a single item taking an exceptionally high value) and the "core" contribution that is spread over many items. Run the better of the following two algorithms:

Tail. Let $O P T$ denote the expected offline optimum value. Whenever we see an item with value at least $2 O P T$, we select it. For item $i$, let $p_{i}=\operatorname{Pr}\left[X_{i} \geq 2 O P T\right]$. We have

$$
O P T \geq 2 O P T \cdot \operatorname{Pr}\left[\exists i: X_{i} \geq 2 O P T\right]=2 O P T \cdot\left(1-\prod\left(1-p_{i}\right)\right) .
$$

Dividing by $O P T$ and rearranging, we get

$$
1 / 2 \leq \prod\left(1-p_{i}\right) \leq e^{-\sum p_{i}},
$$

and thus

$$
\sum p_{i} \leq \ln 2
$$

Therefore the probability that we want to take an item but can't is at most $\ln 2$, so this algorithm achieves at least a $(1-\ln 2)$-fraction of the expected contribution from values greater than $2 O P T$.

Core. Observe that we can safely ignore values less than $O P T / 2 r$, as those can contribute a total of at most $O P T / 2$. Partition all remaining values into $\log r+2$ intervals $[O P T / 2 r, O P T / r], \ldots,[O P T, 2 O P T]$. The expected contribution from the values in each interval is a $\Omega(1 / \log r)$ fraction of the expected offline optimum without values greater than 2OPT. Pick the interval with the largest expected contribution, round down all the values in this interval, and run the algorithm guaranteed by Theorem 2 . This achieves an $\Omega\left(\frac{1}{\log n \cdot \log r}\right)$-fraction of the expected contribution from values less than or equal to $2 O P T$.

The rest of this section is devoted to the proof of Theorem 2 .

\subsection{A Dynamic Potential Function}

A natural approach to solving Downward-Closed PROPHET is the following meta-algorithm: wait for an item with value 1 , and select it if that does not decrease some potential function. In the full version we discuss a few intuitive potential functions and the difficulties that arise in 
analyzing each of them. Here we go directly to the remedy to those obstacles: a "dynamic" potential function.

The basic question we want to ask about a new item is the following: given the realizations we have observed so far and items we have already selected, is it a good idea to select this item? The main challenge is to come up with an analyzable proxy to "good idea". To this end, we consider a restricted prophet who is committed to past decisions -and must also select the current item- but is omniscient about future realizations; we maintain a target value $\tau$, and ask what is the probability over future realizations that the restricted prophet can obtain a solution with value $\tau$. The main novelty in our analysis is that we dynamically update $\tau$. In particular, as the prophet becomes more restricted by accumulating commitments, we compare his solution's value to a lower target $\tau$.

At each iteration, the algorithm maintains a target value $\tau$ and a target probability $\pi ; \pi$ is the probability (over future realizations) that the current restricted prophet beats $\tau$. We say that an item is good if selecting it does not decrease the probability of beating the target value by a factor greater than $n^{2}$, and bad otherwise. Notice that all the bad items together contribute at most a $(1 / n)$-fraction of the probability of beating $\tau$. As we mentioned above, a key ingredient is that $\tau$ is updated dynamically. If the probability of observing a good item with value 1 is too low (less than $1 / 3$ ), we deduct 1 from $\tau$. We show (Lemma 2) that this increases $\pi$ by a factor of at least 2. $\pi$ decreases by an $n^{2}$ factor when we select an item, and increases by a factor of 2 whenever we deduct 1 from $\tau$ : we balance $2 \log n$ deductions for every item the algorithm selects, and this gives the $O(\log n)$ competitive ratio.

So far our algorithm is roughly as follows: set a target value $\tau$; whenever the probability $\pi$ of reaching the target $\tau$ drops below $1 / 3$, decrease $\tau$; if $\pi>1 / 3$, sit and wait for a good item with value 1 - one will arrive with probability at least $1 / 3-o(1)$. There is one more subtlety: what should the algorithm do if all the good items have value 0 ? In other words, what if the probability of observing a good item with value 1 is neither very low nor very close to 1 , say $1 / 2$ or even $1-\frac{1}{\log n}$ ? On one hand, we can't decrease $\tau$ again, because we are no longer guaranteed a significant increase in $\pi$; on the other hand, after, say $\Theta\left(\log ^{2} n\right)$ iterations, we still have a high probability of having an iteration where none of the good items has value 1. (If no good 1's are coming, we don't want the algorithm to wait forever...) Fortunately, there is a simple solution: the algorithm waits for the last good item; if, against the odds, no 1's have yet been observed, the algorithm "hallucinates" that this last item has value 1 , and selects it. In expectation, at most a constant fraction of the items we select will have value 0 , so the competitive ratio is still $O(\log n)$.

\subsection{Formal Description of the Algorithm}

\subsubsection{Notation}

We let $O P T$ denote the expected (offline) optimum. $W$ is the set of items selected so far ( $W$ for "Wins"), and $\ell_{W} \triangleq$ $\max \{i \in W\}$ is the index of the last selected item.

Let $\mathcal{F}$ denote the family of all feasible sets. Similarly, $\mathcal{F}_{W}$ is the family of feasible sets that contain $W$, and $\mathcal{F}_{W+j} \triangleq$ $\mathcal{F}_{W \cup\{j\}}$ is the family of feasible sets that contain $W \cup\{j\}$.
Let $X_{i}$ denote the random value of the $i$-th item. We use $z_{i}$ to refer to the observed realization of $X_{i}$. We let $V\left(\mathcal{F}, X_{[n]}\right) \triangleq \max _{S \in \mathcal{F}} \sum_{i \in S} X_{i}$ denote the value of optimum offline solution (note that this is also a random variable).

Let $\tau=\tau(W)$ be the current target value, and $\pi=$ $\pi(\tau, W)$ denotes the target probability:

$$
\pi(\tau, W) \triangleq \operatorname{Pr}\left[V\left(\mathcal{F}_{W}, X_{[n]}\right)>\tau \mid X_{\left[\ell_{W}\right]}=z_{\left[\ell_{W}\right]}\right] .
$$

We also define $\pi^{j}=\pi^{j}(\tau, W)$ to be the probability of reaching $\tau$, given that $j$ is the next item we select. Formally, we define $\pi^{j}(\tau, W)$ to be equal to:

$$
\operatorname{Pr}\left[V\left(\mathcal{F}_{W+j}, X_{[n]}\right)>\tau \mid X_{[j]}=(z_{\left[\ell_{W}\right]}, \underbrace{0 \ldots 0}_{\ell_{W}+1, \ldots, j-1}, 1)\right] .
$$

We say that a future item is good (and bad otherwise) if $\pi^{j} \geq n^{-2} \cdot \pi$. Finally, $G=G(\tau, \pi, W) \triangleq$ $\left\{j>\ell_{W}: \pi^{j} \geq n^{-2} \cdot \pi\right\} \cap\left(\bigcup_{S \in \mathcal{F}_{W}} S\right)$ is the set of items that are both good and feasible, and $A=A(G) \triangleq$ $\left(\bigvee_{j \in G} X_{j}=1\right)$ is the event that at least one of the good items has value 1 .

\subsubsection{Algorithm}

Initialize $\tau \leftarrow O P T / 2$ and $W \leftarrow \emptyset$.

After each update to $W$, decrease $\tau$ until $\operatorname{Pr}[A] \geq 1 / 3$, or until $|W|>\tau$. When $\operatorname{Pr}[A] \geq 1 / 3$, reveal the values of items until observing a good item (i.e. some $j \in G$ ) with value 1 . When we observe a good item with value 1 , add it to $W$. If we reached the last good item without observing any good items with value 1 , add the last good item to $G$ and subtract 1 from $\tau$. See also pseudocode in Algorithm 1 .

\subsection{Analysis}

\subsubsection{Concentration}

We want to argue that the value of the optimum concentrates around its expectation. Proving concentration for a maximum over an arbitrary family of sets' sums is rather non-trivial. Fortunately, there is a vast literature on concentration bounds for suprema of empirical processes. We use the following inequality due to Ledoux. (It is particularly convenient because the denominator in the exponent depends on the expected supremum rather on absolute bounds on the values each item can take.)

TheOREm 3. [16, Theorem 2.4] There exists some constant $K>0$ such that the following holds. Let $Y_{i}$ 's be independent (but not necessarily identical) random variables in some space $S$; let $\mathcal{C}$ be a countable class of measurable functions $f: S \rightarrow[0,1]$; and let $Z=\sup _{f \in \mathcal{C}} \sum_{i=1}^{n} f\left(Y_{i}\right)$. Then,

$$
\operatorname{Pr}[Z \geq \mathrm{E}[Z]+t] \leq \exp \left(-\frac{t}{K} \cdot \log \left(1+\frac{t}{\mathrm{E}[Z]}\right)\right) .
$$

To make the connection to our setting, let $Y_{i}$ be the vector in $[0,1]^{|\mathcal{F}|}$ whose $S$-th coordinate is $X_{i}$ if $i \in S$, and 0 otherwise. Let $f_{S}\left(Y_{i}\right) \triangleq\left[Y_{i}\right]_{S}$, so $\sum_{i=1}^{n} f_{S}\left(Y_{i}\right)$ is simply the value of set $S$. Let $\mathcal{C} \triangleq\left\{f_{S}\right\}_{S \in \mathcal{F}}$. The above concentration 


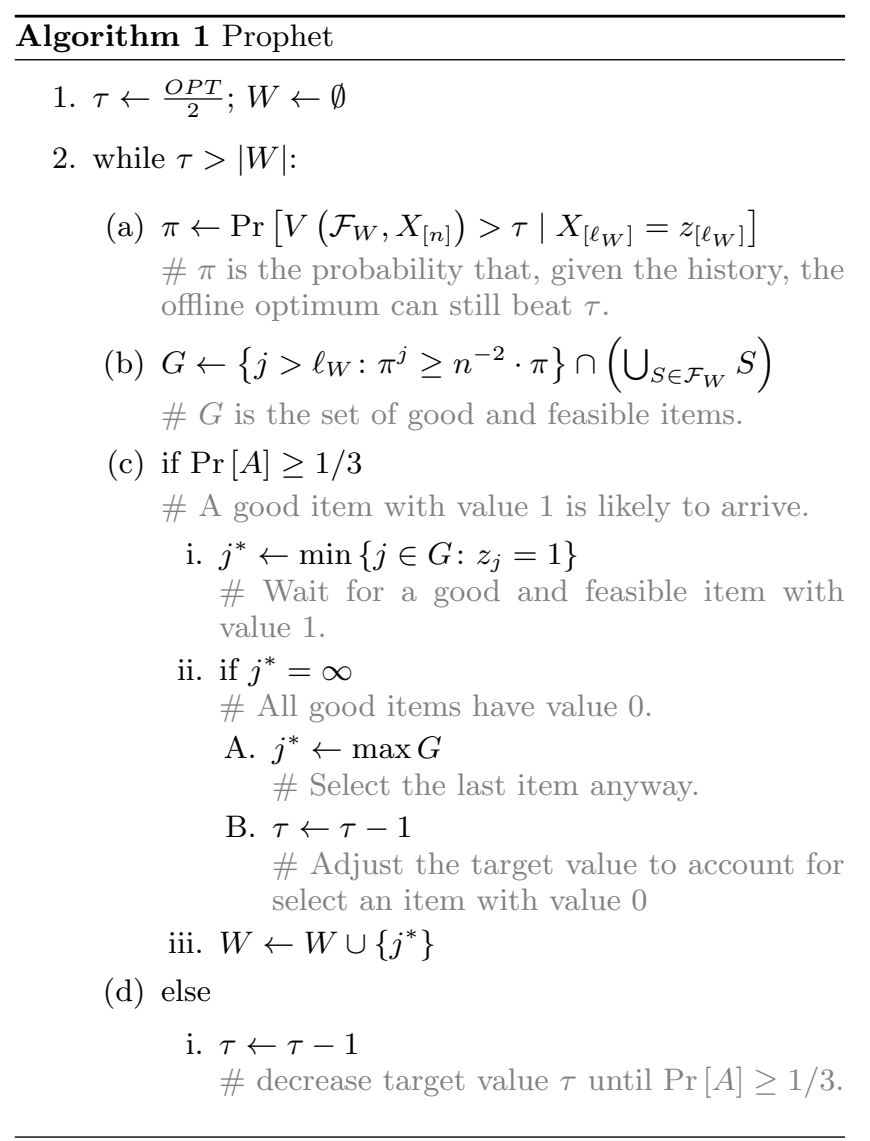

inequality can now be written as

$\operatorname{Pr}\left[V\left(\mathcal{F}, X_{[n]}\right) \geq O P T+t\right] \leq \exp \left(-\frac{t}{K} \cdot \log \left(1+\frac{t}{O P T}\right)\right)$

In fact, we only need the following much weaker lemma. Notice that we can assume without loss of generality that $O P T \geq \log n$; otherwise the trivial greedy algorithm guarantees an expected value of $\Omega(\min \{O P T, 1\})=$ $\Omega(O P T / \log n)$.

Lemma 1. Assume $O P T \geq \Omega(\log n)$. Then,

$$
\operatorname{Pr}\left[V\left(\mathcal{F}, X_{[n]}\right) \geq \frac{O P T}{2}\right]>1 / 4 .
$$

Proof. We have,

$$
O P T=\int_{-O P T}^{\infty} \operatorname{Pr}\left[V\left(\mathcal{F}, X_{[n]}\right) \geq O P T+t\right] d t
$$

which can be decomposed as to integrals over $[-O P T,-O P T / 2],[-O P T / 2, O P T]$, and $[O P T, \infty]$.

The first two integrals can be easily bounded as

$$
\begin{aligned}
\int_{-O P T}^{-O P T / 2} \operatorname{Pr}\left[V\left(\mathcal{F}, X_{[n]}\right) \geq O P T+t\right] d t & \leq \int_{-O P T}^{-O P T / 2} 1 \cdot d t \\
& \leq \frac{O P T}{2}
\end{aligned}
$$

and

$$
\begin{aligned}
\int_{-O P T / 2}^{O P T} \operatorname{Pr} & {\left[V\left(\mathcal{F}, X_{[n]}\right) \geq O P T+t\right] d t } \\
\leq & \int_{-O P T / 2}^{O P T} \operatorname{Pr}\left[V\left(\mathcal{F}, X_{[n]}\right) \geq O P T / 2\right] d t \\
\leq & \frac{3 O P T}{2} \cdot \operatorname{Pr}\left[V\left(\mathcal{F}, X_{[n]}\right)>\frac{O P T}{2}\right] .
\end{aligned}
$$

For the third integral we use the concentration bound (1):

$$
\begin{aligned}
\int_{O P T}^{\infty} \operatorname{Pr}[V & \left.\left(\mathcal{F}, X_{[n]}\right) \geq O P T+t\right] d t \\
& \leq \int_{O P T}^{\infty} \exp \left(-\frac{t}{K} \cdot \log \left(1+\frac{t}{O P T}\right)\right) d t \\
& \leq \int_{O P T}^{\infty} \exp \left(-\frac{t}{K}\right) d t \\
& =\left[K e^{-t / K}\right]_{O P T}^{\infty} \\
& =K \cdot e^{-O P T / K},
\end{aligned}
$$

which is negligible since $O P T=\omega(1)$.

Plugging into (2), we have that $O P T$ is at most:

$$
\frac{O P T}{2}+\frac{3 O P T}{2} \cdot \operatorname{Pr}\left[V\left(\mathcal{F}, X_{[n]}\right)>\frac{O P T}{2}\right]+o(1)
$$

and after rearranging we get

$$
\operatorname{Pr}\left[V\left(\mathcal{F}, X_{[n]}\right)>\frac{O P T}{2}\right] \geq 1 / 3-o(1) .
$$

\subsubsection{Main Lemma}

LEMma 2. At any point during the run of the algorithm, if $\operatorname{Pr}[A] \leq 1 / 3$, then subtracting 1 from $\tau$ doubles $\pi$; i.e.

$$
\begin{aligned}
& \operatorname{Pr}\left[V\left(\mathcal{F}_{W}, X_{[n]}\right)>\tau-1 \mid X_{\left[\ell_{W}\right]}=z_{\left[\ell_{W}\right]}\right] \\
& \quad \geq 2 \operatorname{Pr}\left[V\left(\mathcal{F}_{W}, X_{[n]}\right)>\tau \mid X_{\left[\ell_{W}\right]}=z_{\left[\ell_{W}\right]}\right] .
\end{aligned}
$$

Proof. We first claim that the probability that the optimum solution (conditioned on the items $W$ we already selected and the realizations $z_{\left[\ell_{W}\right]}$ we have already seen) reaches $\tau$ without selecting any items from $G$ is negligible with respect to the total probability of reaching $\tau$. For each $k \notin G$, we have, by definition of $G$,

$$
\begin{array}{r}
\underbrace{\operatorname{Pr}\left[V\left(\mathcal{F}_{W+k}, X_{[n]}\right)>\tau \mid X_{[k]}=\left(z_{\left[\ell_{W}\right]}, 0 \ldots 0,1\right)\right]}_{\pi^{k}} \\
<n^{-2} \cdot \underbrace{\operatorname{Pr}\left[V\left(\mathcal{F}_{W}, X_{[n]}\right)>\tau \mid X_{\left[\ell_{W}\right]}=z_{\left[\ell_{W}\right]}\right]}_{\pi} .
\end{array}
$$

However, if $X_{j}=0$ for all $j \in G$ (i.e. if event $A$ does not happen), the first item that contributes to the optimum solution has to be some $k \notin G$. Thus the probability that it still reaches $\tau$ is very small:

$$
\begin{gathered}
\operatorname{Pr}\left[\left(V\left(\mathcal{F}_{W}, X_{[n]}\right)>\tau\right) \wedge(\neg A) \mid\left(X_{\left[\ell_{W}\right]}=z_{\left[\ell_{W}\right]}\right)\right] \\
\leq \sum_{k \notin G} \operatorname{Pr}\left[\left(\mathcal{F}_{W+k}, X_{[n]}\right)>\tau \mid \ldots\right. \\
\left.\quad \ldots X_{[k]}=\left(z_{\left[\ell_{W}\right]}, 0 \ldots 0,1\right)\right] \\
\leq n^{-1} \cdot \operatorname{Pr}\left[V\left(\mathcal{F}_{W}, X_{[n]}\right)>\tau \mid X_{\left[\ell_{W}\right]}=z_{\left[\ell_{W}\right]}\right] .
\end{gathered}
$$


In particular, most of the probability of reaching $\tau$ comes from the event $A$ (i.e. $X_{j}=1$ for some $j \in G$ ):

$$
\begin{gathered}
\operatorname{Pr}\left[\left(V\left(\mathcal{F}_{W}, X_{[n]}\right)>\tau\right) \wedge A \mid X_{\left[\ell_{W}\right]}=z_{\left[\ell_{W}\right]}\right] \\
\geq\left(1-n^{-1}\right) \operatorname{Pr}\left[V\left(\mathcal{F}_{W}, X_{[n]}\right)>\tau \mid \ldots\right. \\
\left.\ldots X_{\left[\ell_{W}\right]}=z_{\left[\ell_{W}\right]}\right] .
\end{gathered}
$$

Inconveniently, this does not guarantee that $\operatorname{Pr}[A]$ is large, because the probability of reaching $\tau$ may be very small to begin with, and those events are not independent. Instead, rewrite the LHS of the above equation as

$$
\begin{aligned}
\operatorname{Pr} & {\left[\left(V\left(\mathcal{F}_{W}, X_{[n]}\right)>\tau\right) \wedge A \mid X_{\left[\ell_{W}\right]}=z_{\left[\ell_{W}\right]}\right] } \\
& \underbrace{\operatorname{Pr}[A]}_{\leq 1 / 3} \operatorname{Pr}\left[V\left(\mathcal{F}_{W}, X_{[n]}\right)>\tau \mid\left(X_{\left[\ell_{W}\right]}=z_{\left[\ell_{W}\right]}\right) \wedge A\right]
\end{aligned}
$$

By the premise, this implies a strong lower bound on the second term on the RHS. Finally, we claim that conditioning on $A$ can contribute at most 1 to the optimum $V\left(\mathcal{F}_{w}, X_{[n]}\right)$. Assuming this claim, the proof of the lemma is complete:

$$
\begin{aligned}
\operatorname{Pr} & {\left[V\left(\mathcal{F}_{W}, X_{[n]}\right)>\tau-1 \mid X_{\left[\ell_{W}\right]}=z_{\left[\ell_{W}\right]}\right] } \\
& \geq \operatorname{Pr}\left[V\left(\mathcal{F}_{W}, X_{[n]}\right)>\tau \mid\left(X_{\left[\ell_{W}\right]}=z_{\left[\ell_{W}\right]}\right) \wedge A\right] \\
& \geq(3-o(1)) \operatorname{Pr}\left[V\left(\mathcal{F}_{W}, X_{[n]}\right)>\tau \mid X_{\left[\ell_{W}\right]}=z_{\left[\ell_{W}\right]}\right] .
\end{aligned}
$$

It remains to prove (3). Rewrite $A$ as a disjoint union of the events $A^{j} \triangleq\left(X_{j}=1 \bigwedge_{\substack{i \in j \\ i \in G}} X_{i}=0\right)$, i.e. $A^{j}$ is the event that $j$ is the first good item with value 1 . Let $\pi_{A}$ denote the probability of beating the target value conditioning on the history and event $A$, and analogously for $\pi_{A^{j}}$ :

$$
\begin{aligned}
\pi_{A} & \triangleq \operatorname{Pr}\left[V\left(\mathcal{F}_{W}, X_{[n]}\right)>\tau \mid X_{\left[\ell_{W}\right]}=z_{\left[\ell_{W}\right]} \wedge A\right] \\
\pi_{A^{j}} & \triangleq \operatorname{Pr}\left[V\left(\mathcal{F}_{W}, X_{[n]}\right)>\tau \mid X_{\left[\ell_{W}\right]}=z_{\left[\ell_{W}\right]} \wedge A^{j}\right] .
\end{aligned}
$$

Let $B^{j} \triangleq\left(X_{j}=0 \bigwedge_{\substack{i<j \\ i \in G}} X_{i}=0\right)$ be the event that neither the $j$-th item nor any of the preceding good items have value 1 . Changing the value of $X_{j}$ can decrease the final value of the solution by at most 1 . Thus, for every $j$,

$$
\begin{aligned}
\pi_{A^{j}} & \leq \operatorname{Pr}\left[V\left(\mathcal{F}_{W}, X_{[n]}\right)>\tau-1 \mid X_{\left[\ell_{W}\right]}=z_{\left[\ell_{W}\right]} \wedge B^{j}\right] \\
& \leq \operatorname{Pr}\left[V\left(\mathcal{F}_{W}, X_{[n]}\right)>\tau-1 \mid X_{\left[\ell_{W}\right]}=z_{\left[\ell_{W}\right]}\right],
\end{aligned}
$$

where the last inequality follows because conditioning on $B^{j}$ can only decrease the probability of reaching the target value.

Finally, since $A$ is a disjoint union of the $A^{j}$ 's, it follows that $\pi_{A}$ is a convex combination of the $\pi_{A^{j}}$ 's. Therefore,

$$
\begin{aligned}
& \operatorname{Pr}\left[V\left(\mathcal{F}_{W}, X_{[n]}\right)>\tau \mid X_{\left[\ell_{W}\right]}=z_{\left[\ell_{W}\right]} \wedge A\right]=\pi_{A} \\
& \leq \operatorname{Pr}\left[V\left(\mathcal{F}_{W}, X_{[n]}\right)>\tau-1 \mid X_{\left[\ell_{W}\right]}=z_{\left[\ell_{W}\right]}\right] .
\end{aligned}
$$

\subsubsection{Putting It All Together}

Lemma 3. At any point during the run of the algorithm,

$$
\tau \geq \frac{O P T}{2}-(2 \log n+1) \cdot|W|-2
$$

Proof. We prove by induction that at any point during the run of the algorithm,

$$
\log \pi \geq-2-(2 \log n+1) \cdot|W|+\left(\frac{O P T}{2}-\tau\right) .
$$

After initialization, $\log \pi \geq-2$ by Lemma 1 . By definition of $G$, whenever we add an item to $W$, we decrease $\log \pi$ by at $\operatorname{most} 2 \log n$ - hence the $2 \log n \cdot|W|$ term. Notice that when the algorithm "hallucinates" a 1 , we also decrease $\tau$ by 1 to correct for the hallucination - at any point during the run of the algorithm, this has happened at most $|W|$ times. Recall that we may also decrease $\tau$ in the last line of Algorithm 1 (in order to increase $\pi$ ); whenever we do this, $\tau$ decreases by 1 , but $\pi$ doubles (by Lemma 2 ), so $\log \pi$ increases by 1 , and Inequality (4) is preserved.

Finally, since $\pi$ is a probability, we always maintain $\log \pi \leq 0$.

We are now ready to complete the proof of Theorem 2 .

Proof of Theorem 2. The algorithm always terminates after at most $O(O P T)$ decreases to the value of $\tau$. By Lemma 3, when the algorithm terminates, we have $|W| \geq \tau \geq \frac{O P T}{2}-(2 \log n+1) \cdot|W|-2$, and therefore in particular $|W| \geq \frac{O P T-4}{4 \log n+4}$.

Finally, recall that sometimes the algorithm "hallucinates" good realizations, i.e. for some items $i \in W$ that we select, $X_{i}=0$. However, each time we add an item, the probability that we add a zero-value item is at most $2 / 3$ (by the condition $\operatorname{Pr}[A]>1 / 3)$. Therefore in expectation the value of the algorithm is at least $|W| / 3$.

\section{SECRETARY}

We now discuss our algorithm for the DownwARDClosed Secretary problem. Recall that as we mentioned earlier, it is very different from our algorithm for Downward-Closed Prophet.

Definition 2. (Downward-Closed Secretary) Consider $n$ items with values $\left\{y_{i}\right\}$ and an arbitrary downward-closed set system $\mathcal{F}$ over the items; both $\left\{y_{i}\right\}$ and $\mathcal{F}$ are adversarially chosen. The algorithm receives as input $n$ (but not $\mathcal{F}$ or $\left\{y_{i}\right\}$ ). The items arrive in a uniformly random order. Initialize $W$ as the empty set. When item $i$ arrives, the algorithm observes its value and all feasible sets it forms with items that have previously arrived. The algorithm then decides (immediately and irrevocably) whether to add $i$ to the set $W$, subject to the constraint that $W$ remains a feasible set in $\mathcal{F}$. The goal is to maximize the sum of values of items in $W$.

We remark that our analysis for the $\{0,1\}$-valued case does not require a uniformly random arrival order. Rather, partition the time intervals into pairs $\{j, n / 2+j\}$; the items can be assigned arbitrarily to pairs of arriving times, and we only require that the choice of which of the two items arrives at time $j$ and which at time $n / 2+j$ is random.

TheOREM 4. When $y_{i} \in\{0,1\}$ for all $i$, there is a deterministic algorithm for DOWNWARD-CLOSED SECRETARY that achieves a competitive ratio of $O(\log n)$.

Let $r$ denote the maximum cardinality of a feasible set $S \in$ $\mathcal{F}$.

Corollary 3. For general valuations, there is a randomized algorithm for DownWARD-ClOSED SECRETARY that achieves a competitive ratio of $O(\log n \cdot \log r)$.

Proof of Corollary 3 from Theorem 4. Run the classic secretary algorithm over the first $n / 2$ items. With 
constant probability the algorithm selects the item with the largest value, which we denote by $M$.

Also, with constant probability the algorithm sees the item with the largest value too early and does not select it. Assume that this is the case. Since we obtained expected value of $\Omega(M)$ on the first $n / 2$ items we can, without loss of generality, ignore values less than $M / r$. Partition all remaining values into $\log r$ intervalbuckets $[M / r, 2 M / r], \ldots,[M / 2, M]$; in expectation, each contributes a $(1 / \log r)$-fraction of the optimum. Choose a bucket at random, round all the values in it to 1 and set the rest to 0 . Finally, run the $O(\log n)$-competitive algorithm from Theorem 4 on the last $n / 2$ items. (We remark that the choice of bucket can be derandomized using the random order of the first $n / 2$ items.)

The rest of this section is devoted to the proof of Theorem 4 .

\subsection{Secretary Algorithm}

We run a greedy algorithm over a sliding window of size $n / 2$. At first, we have a fully offline solution (none of which we actually select) over the first $n / 2$ items. We gradually transform it into a real online solution over the last $n / 2$ items.

Intuitively, we test how well each item interacts with the previous $n / 2$ items to estimate how well it would interact with future items. The main challenge is that we reuse the same randomness from the previous items over and over, and we may overfit our choices to the past. We overcome this by showing that the probability that the previous items do not represent the future is very small - so small that we can take a union bound over all the potential actions of our algorithm. Roughly speaking, our main argument only uses 1 bit of randomness for each item in the optimal offline solution (does it arrive in one of the first $n / 2$ time periods?), so we cannot expect any concentration inequality to bound the deviations to probability less than $2^{-O P T}$. On the other hand, if our algorithm selects $\tau$ items, we have to take a union bound over $\left(\begin{array}{l}n \\ \tau\end{array}\right)$ sets, so we want $2^{-O P T} \cdot\left(\begin{array}{l}n \\ \tau\end{array}\right) \ll$ 1. Taking $\tau \approx O P T / \log n$ gives the promised competitive ratio.

Notation. For any set $T$, we let $V(\mathcal{F} ; T)$ denote the value of the offline optimum solution restricted to $T$. We let $\mathcal{F}_{W}$ denote the feasible sets that contain $W$, and finally $V\left(\mathcal{F}_{W} ; T\right)$ denotes the value of the offline optimum among feasible sets that contain $W$ and are contained in $T$. We use $\sigma$ to denote the order of arrivals; in particular, $\sigma_{j}$ is the item that arrives in the $j$-th time period.

Algorithm. In the exploration phase, we observe the first $n / 2$ items and add none of them to $W$; we initialize $U$ as the set of those items (notice that $U$ needs not be a feasible set), and set our target value to $\tau \triangleq V(\mathcal{F} ; U) / 500 \log n$. In the exploitation phase, before observing the $(n / 2+j)$ th item, we "forget" the $j$-th item, i.e. we permanently remove $\sigma_{j}$ from $U$. Then, we add the $\sigma_{n / 2+j}$ to $W$ iff $V\left(\mathcal{F}_{W \cup\left\{\sigma_{n / 2+j}\right\}} ; U \cup W \cup\left\{\sigma_{n / 2+j}\right\}\right)>V\left(\mathcal{F}_{W} ; U \cup W\right)$. Continue until $|W|=\tau$ or until all items have been revealed. (Of course, aborting when $|W|=\tau$ can only hurt the expected value of the solution, but this simplifies the analysis by restricting the loss from taking a union bound.) See also pseudocode in Algorithm 2.

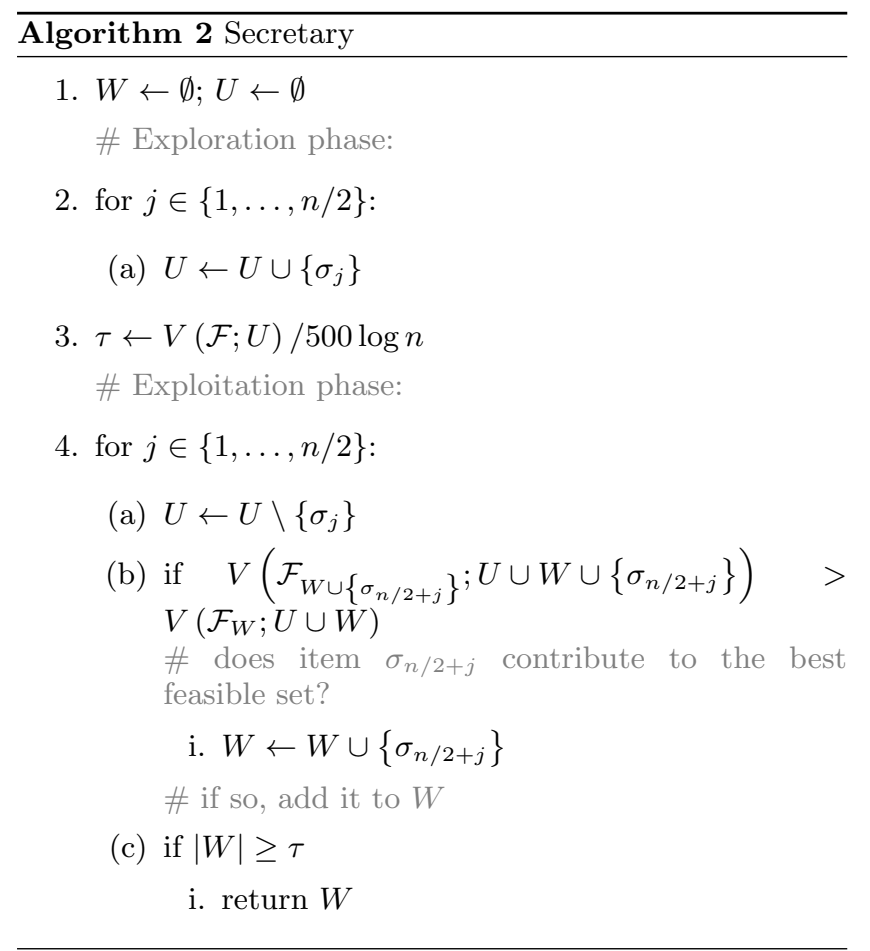

\subsection{Analysis}

Setup. Our analysis compares the number of times the value of the current solution decreases when we forget $\sigma_{j}$ ("bad" events), with the number of "good" events, where the value of the current solution increases when we add $\sigma_{n / 2+j}$.

Let $U_{j}$ denote the set $U$ after we remove the $j$-th item, i.e. $U_{j} \triangleq\left\{\sigma_{j+1}, \ldots, \sigma_{n / 2}\right\}$. We henceforth let $W$ refer to the set returned by the algorithm; for intermediate values, we let $W_{j}$ denote the set $W$ before we consider item $\sigma_{n / 2+j}$, i.e. $W_{j} \triangleq W \cap\left\{\sigma_{n / 2+1}, \ldots, \sigma_{n / 2+j-1}\right\}$.

We want to take a union bound over all choices the algorithm could have made. Fix any choice of feasible set $\widehat{W}$ of size at most $\tau$ and a choice $\left.\widehat{\sigma}\right|_{\widehat{W}}$ of arrival times for those items. For any $j$, let $\widehat{W}_{j} \triangleq \widehat{W} \cap\left\{\widehat{\sigma}_{n / 2+1}, \ldots, \widehat{\sigma}_{n / 2+j-1}\right\}$. For each $j$, let $B_{j}\left(\widehat{W},\left(\left.\widehat{\sigma}\right|_{\widehat{W}}\right)\right)$ ( $B$ for "bad") be the event that forgetting $\sigma_{j}$ decreases the value of the current solution, i.e. $B_{j}\left(\widehat{W},\left(\left.\widehat{\sigma}\right|_{\widehat{W}}\right)\right)=1$ iff

$$
V\left(\mathcal{F}_{\widehat{W}_{j} \cup\left\{\sigma_{j}\right\}} ; U_{j} \cup \widehat{W}_{j} \cup\left\{\sigma_{j}\right\}\right)>V\left(\mathcal{F}_{\widehat{W}_{j}} ; U_{j} \cup \widehat{W}_{j}\right),
$$

and otherwise $B_{j}\left(\widehat{W},\left(\left.\widehat{\sigma}\right|_{\widehat{W}}\right)\right)=0$.

Similarly, let $G_{j}\left(\widehat{W},\left(\left.\widehat{\sigma}\right|_{\widehat{W}}\right)\right)(G$ for "good") denote the event that adding $\sigma_{n / 2+j}$ increases the value of the current solution: $G_{j}\left(\widehat{W},\left(\left.\widehat{\sigma}\right|_{\widehat{W}}\right)\right)=1 \mathrm{iff}$

$$
\begin{gathered}
V\left(\mathcal{F}_{\widehat{W}_{j} \cup\left\{\sigma_{n / 2+j}\right\}} ; U_{j} \cup \widehat{W}_{j} \cup\left\{\sigma_{n / 2+j}\right\}\right)> \\
V\left(\mathcal{F}_{\widehat{W}_{j}} ; U_{j} \cup \widehat{W}_{j}\right),
\end{gathered}
$$

and $G_{j}\left(\widehat{W},\left(\left.\widehat{\sigma}\right|_{\widehat{W}}\right)\right)=0$ otherwise. 
By symmetry, we have that

$$
\operatorname{Pr}\left[B_{j}\left(\widehat{W},\left(\left.\widehat{\sigma}\right|_{\widehat{W}}\right)\right)\right]=\operatorname{Pr}\left[G_{j}\left(\widehat{W},\left(\left.\widehat{\sigma}\right|_{\widehat{W}}\right)\right)\right] .
$$

When the choice of $\left(\widehat{W},\left(\left.\widehat{\sigma}\right|_{\widehat{W}}\right)\right)$ is clear from the context, we will simply write $B_{j}$ and $G_{j}$; we abuse notation and also use $B_{j}$ and $G_{j}$ to denote the corresponding indicator random variables. Notice also that we do not require that $\sigma$ agrees with $\widehat{\sigma}$, and the unions in the definitions of $B_{j}$ and $G_{j}$ may not be disjoint unions.

We will of course be interested in the special case where $\widehat{W}=W$ and $\widehat{\sigma}$ agrees with $\sigma$. Notice that as long as we have not reached the target value $\tau$, the algorithm selects every $j$ for which the good event $G_{j}(W,(\sigma \mid W))$ occurs. Thus, the value of our solution is

$$
|W|=\min \left\{\tau, \sum G_{j}\left(W,\left(\sigma \mid{ }_{W}\right)\right)\right\} .
$$

Furthermore, the value of the current solution decreases $\sum B_{j}\left(W,\left(\left.\sigma\right|_{W}\right)\right)$ times, and increases $|W|$ times. At the beginning, the value is $V\left(\mathcal{F} ; U_{0}\right)$ and at the end it is $|W|$. Canceling $|W|$ from both sides of the equation, we get

$$
V\left(\mathcal{F} ; U_{0}\right)=\sum B_{j}\left(W,\left(\left.\sigma\right|_{W}\right)\right) .
$$

Our goal is henceforth to lower bound $\sum G_{j}$ in terms of $\sum B_{j}$ for all choices of $\widehat{W}$ and $\left(\left.\widehat{\sigma}\right|_{\widehat{W}}\right)$ simultaneously. For the special case where $\widehat{W}=W$ and $\left(\left.\widehat{\sigma}\right|_{\widehat{W}}\right)=\left(\left.\sigma\right|_{W}\right)$, this will in particular imply a lower bound on $|W|$ in terms of $V\left(\mathcal{F} ; U_{0}\right)$.

Concentration. We will use the following martingale version of Bennett's inequality. (The main advantage over the more popular Azuma's inequality is that this theorem depends on the variance rather than an absolute bound on the values each variable can take.)

ThEOREM 5. [e.g. [9]] Let $(\Omega, \mathcal{H}, \mu)$ be a probability triple, with $\mathcal{H}_{0} \subseteq \mathcal{H}_{1} \subseteq \ldots$ an increasing sequence of sub- $\sigma$-fields of $\mathcal{H}$. For each $k=1,2, \ldots$ let $X_{k}$ be an $\mathcal{H}_{k}$-measurable random variable satisfying $\left|X_{k}\right| \leq 1$ and $\mathrm{E}\left[X_{k} \mid \mathcal{H}_{k-1}\right]=0$. Let $S_{n} \triangleq \sum_{k=1}^{n} X_{k}$ and $T_{n} \triangleq$ $\sum_{k=1}^{n} \mathrm{E}\left[X_{k}^{2} \mid \mathcal{H}_{k-1}\right]$. Then, for every $a, b>0$,

$$
\operatorname{Pr}\left[\left(S_{n} \geq a\right) \wedge\left(T_{n} \leq b\right)\right] \leq \exp \left(\frac{-a^{2}}{2(a+b)}\right) .
$$

Main Argument. For the analysis, we reveal the items in a special order: at step 0 we reveal the pairs $\left\{\sigma_{j}, \sigma_{n / 2+j}\right\}$ unordered, i.e. we reveal all the information up to the last $n / 2$ random bits which determine which item arrives at time $j$, and which at time $n / 2+j$. (This information corresponds to $\mathcal{H}_{0}$ in Theorem 5.) In fact, those pairs can be chosen adversarially. Then, we reveal the pairs in reverse order, beginning with $\left(\sigma_{n / 2}, \sigma_{n}\right)$. (The knowledge of the last $k$ pairs corresponds to $\mathcal{H}_{k}$.)

Fix some choice of $\widehat{W}$ and $\left.\widehat{\sigma}\right|_{\widehat{W}}$. For $k=1, \ldots, n / 2$, let $X_{k} \triangleq B_{n / 2-k+1}-G_{n / 2-k+1}$. Since we fixed $\widehat{W}_{j}$ and $\left(\left.\widehat{\sigma}\right|_{\widehat{W}_{j}}\right)$ in advance, (5) implies that the $X_{k}$ 's satisfy $\mathrm{E}\left[X_{k} \mid \mathcal{H}_{k-1}\right]=$ 0 as required in the premise of Theorem 5 .

By definition, $S_{n}=\sum\left(B_{j}-G_{j}\right)$. Also, for any $k$ we have that $\mathrm{E}\left[X_{k}^{2} \mid \mathcal{H}_{k-1}\right]=1$ if (exactly) one of $\left\{\sigma_{n / 2-k+1}, \sigma_{n-k+1}\right\}$ affects the value of the current solution, and $\mathrm{E}\left[X_{k}^{2} \mid \mathcal{H}_{k-1}\right]=0$ otherwise. Thus

$$
T_{n} \leq \sum\left(B_{j}+G_{j}\right) \leq V(\mathcal{F} ;[n])+\sum G_{j},
$$

where the second inequality follows from (7); in particular, if $\sum B_{j} \geq \sum G_{j}$, we have that $T_{n} \leq 2 V(\mathcal{F} ;[n])$. Theorem 5 now gives

$$
\begin{aligned}
& \operatorname{Pr}\left[\sum\left(B_{j}-G_{j}\right) \geq V(\mathcal{F} ;[n]) / 6\right] \\
& \quad=\operatorname{Pr}\left[\left(S_{n} \geq V(\mathcal{F} ;[n]) / 6\right) \wedge\left(T_{n} \leq 2 V(\mathcal{F} ;[n])\right)\right] \\
& \quad \leq \exp \left(\frac{-V(\mathcal{F} ;[n])}{156}\right) .
\end{aligned}
$$

Taking union bound over all $\left(\begin{array}{l}n \\ \tau\end{array}\right)^{2} \leq n^{2 \tau}$ choices of $\widehat{W}$ and $\left.\widehat{\sigma}\right|_{\widehat{W}}$, we get that with high probability, for all of those choices simultaneously $\sum\left(B_{j}-G_{j}\right) \leq V(\mathcal{F} ;[n]) / 6$. In particular, with high probability,

$$
\sum G_{j}\left(W,\left(\left.\sigma\right|_{W}\right)\right) \geq V\left(\mathcal{F} ; U_{0}\right)-V(\mathcal{F} ;[n]) / 6 .
$$

Furthermore, with probability at least $1 / 2$,

$$
V\left(\mathcal{F} ; U_{0}\right) \geq V(\mathcal{F} ;[n]) / 2,
$$

in which case our algorithm's solution has value at least $\tau \geq V(\mathcal{F} ;[n]) / 1000 \log n$.

\section{NON-MONOTONE FEASIBILITY CON- STRAINTS}

Let Non-monotone Prophet be the analogous variant of Downward-Closed Prophet when the feasibility constraint is not even guaranteed to be downward-closed. Since the feasibility constraint is non-monotone, let us explicitly assume that all item values are non-negative.

THEOREM 6. The competitive ratio for Non-MONOTONE Prophet with non-negative values is $\Theta(n)$.

Proof. Our algorithm simply selects any feasible set that contains the item with the largest expected contribution. Observe that this achieves at least $O P T / n$ in expectation.

We now prove an $\Omega(n)$ lower bound on the competitive ratio. For every $i \in[n / 2]$, the set $\{i, n / 2+i\}$ is feasible (and these are the only feasible sets). Also, let $X_{i}=0$ always, and $X_{n / 2+i}=\left\{\begin{array}{ll}1 & \text { w.p. } 1 / n \\ 0 & \text { otherwise }\end{array}\right.$. Any online algorithm must commit to some $\{i, n / 2+i\}$ before observing any of the $X_{n / 2+i}$ 's, and thus has expected value of $1 / n$. The offline optimum, on the other hand, is $1-(1-1 / n)^{n} \approx 1-1 / e$.

For the Secretary Problem, there are several ways to generalize to non-monotone feasibility constraints. For example, we could assume that the online algorithm knows in advance the entire set system (with or without the arrival times, but certainly without the values). Alternatively, the online algorithm could have an oracle to queries of the form "is $S$ a subset of a feasible set?". The following Theorem holds with respect to either definition.

THEOREM 7. The competitive ratio for Non-MONOTONE SECRETARY is at most $n$, and at least $\Omega\left(n / \log ^{*} n\right)$. 
Proof. Our algorithm simply selects the first item and completes any feasible set that contains it. Since it is a uniformly random item, its value is at least $O P T / n$.

For the lower bound, we instantiate the Hadamard Code with block length $n / 2$. Recall that it has $n / 2$ codewords and the distance between every two codewords is $n / 4$. Let $w_{i} \subseteq[n / 2]$ denote the $i$-th codeword, and consider the set $S_{i} \triangleq w_{i} \cup\{n / 2+i\} \subset[n]$; let the $S_{i}$ 's be the feasible sets. Set $X_{n / 2+i^{*}}=1$ for some $i^{*}$, and let all other items have value 0 . Call $w_{i^{*}}$ the good codeword, and $\left(n / 2+i^{*}\right)$ is the index of the good item.

In order to achieve value 1 , the online algorithm's selections must be consistent with the good codeword when the good item arrives. By symmetry, for each $i$ such that the online algorithm's selection is consistent with $w_{i}$ when item $(n / 2+i)$ arrives, it has probability $1 / n$ of obtaining value 1 . The expected value of the online algorithm is therefore proportional to the number of consistent codewords. Let $k$ be a sufficiently large constant (e.g. $k=100$ ). We show that, in expectation, the algorithm is consistent with $O(1)$ codewords whose items arrive in time periods $(k \log n, n], O(1)$ codewords whose items arrive in time periods $(k \log \log n, k \log n],(k \log \log \log n, k \log \log n]$, etc.. In total the algorithm is consistent with $O\left(\log ^{*} n\right)$ codewords, so its expected value is $O\left(\log ^{*} n / n\right)$, as opposed to value 1 obtained by the offline algorithm.

With high probability, every two codewords disagree after $k \log n$ time periods; i.e. for every two sets $S_{i}, S_{j}$, at least one of the first $k \log n$ items is contained in one and not in the other. Thus after $k \log n$ time periods, the online algorithm must commit to one subset $S_{i}$, so it only wins if $(n / 2+i)$ is the good item. In particular, for any choices an online algorithm may make on the first $k \log n$ items, its expected value from the items that come in time periods $(k \log n, n]$ is $O(1 / n)$.

Similarly, consider the $O(\log n)$ codewords that correspond to items that arrive in time periods $(k \log \log n, k \log n] . \quad$ After $k \log \log n$ codewords, we expect that every two of those $O(\log n)$ codewords disagree on some time period. Thus any online algorithm can obtain expected value at most $O(1 / n)$ from items that arrive in time periods $(k \log \log n, k \log n]$. Same for $(k \log \log \log n, k \log \log n]$, etc.

\section{ACKNOWLEDGMENTS}

I thank Tselil Schramm and anonymous reviewers for comments on earlier drafts. I am also grateful to Sid Barman, Jonathan Hermon, and James Lee for fascinating discussions about concentration bounds. Most importantly, I thank Moshe Babaioff and Bobby Kleinberg for insightful suggestions; in particular, I thank Kleinberg for proposing this problem.

\section{REFERENCES}

[1] P. D. Azar, R. Kleinberg, and S. M. Weinberg. Prophet inequalities with limited information. In Proceedings of the Twenty-Fifth Annual ACM-SIAM Symposium on Discrete Algorithms, SODA 2014, Portland, Oregon, USA, January 5-7, 2014, pages 1358-1377, 2014.

[2] M. Babaioff, N. Immorlica, D. Kempe, and R. Kleinberg. Online auctions and generalized secretary problems. SIGecom Exch., 7(2):7:1-7:11, June 2008.

[3] M. Babaioff, N. Immorlica, and R. Kleinberg. Matroids, secretary problems, and online mechanisms. In Proceedings of the Eighteenth Annual ACM-SIAM Symposium on Discrete Algorithms, SODA 2007, New Orleans, Louisiana, USA, January 7-9, 2007, pages 434-443, 2007.

[4] M. Dinitz. Recent advances on the matroid secretary problem. SIGACT News, 44(2):126-142, 2013.

[5] P. Dütting and R. Kleinberg. Polymatroid prophet inequalities. In Algorithms - ESA 2015 - 23rd Annual European Symposium, Patras, Greece, September 14-16, 2015, Proceedings, pages 437-449, 2015.

[6] E. B. Dynkin. The optimum choice of the instant for stopping a markov process. Sov. Math. Dokl., 1963.

[7] M. Feldman, O. Svensson, and R. Zenklusen. A simple $O(\log \log ($ rank $))$-competitive algorithm for the matroid secretary problem. In Proceedings of the Twenty-Sixth Annual ACM-SIAM Symposium on Discrete Algorithms, SODA 2015, San Diego, CA, USA, January 4-6, 2015, pages 1189-1201, 2015.

[8] M. Feldman and R. Zenklusen. The submodular secretary problem goes linear. CoRR, abs/1507.08384, 2015.

[9] D. A. Freedman. On tail probabilities for martingales. Ann. Probab., 3(1):100-118, 021975.

[10] M. T. Hajiaghayi, R. D. Kleinberg, and T. Sandholm. Automated online mechanism design and prophet inequalities. In Proceedings of the Twenty-Second AAAI Conference on Artificial Intelligence, July 22-26, 2007, Vancouver, British Columbia, Canada, pages 58-65, 2007.

[11] T. Kesselheim, R. D. Kleinberg, and R. Niazadeh. Secretary problems with non-uniform arrival order. In Proceedings of the Forty-Seventh Annual ACM on Symposium on Theory of Computing, STOC 2015, Portland, OR, USA, June 14-17, 2015, pages 879-888, 2015.

[12] R. Kleinberg and S. M. Weinberg. Matroid prophet inequalities. In Proceedings of the 44th Symposium on Theory of Computing Conference, STOC 2012, New York, NY, USA, May 19 - 22, 2012, pages 123-136, 2012.

[13] R. D. Kleinberg, May 2015. Private Communication.

[14] U. Krengel and L. Sucheston. Semiamarts and finite values. Bull. Amer. Math. Soc., 83(4):745-747, 07 1977.

[15] O. Lachish. O(log $\log$ rank) competitive ratio for the matroid secretary problem. In 55th IEEE Annual Symposium on Foundations of Computer Science, FOCS 2014, Philadelphia, PA, USA, October 18-21, 2014, pages 326-335, 2014.

[16] M. Ledoux. On Talagrand's deviation inequalities for product measures. ESAIM: Probability and Statistics, 1:63-87, 1997.

[17] S. Vardi. The returning secretary. In 32nd International Symposium on Theoretical Aspects of Computer Science, STACS 2015, March 4-7, 2015, Garching, Germany, pages 716-729, 2015. 


\section{APPENDIX}

A. AN $\Omega\left(\frac{\log N}{\log \log N}\right)$ LOWER BOUND

For completeness, we briefly sketch an $\Omega(\log n / \log \log n)$ lower bound due to [3] for the competitive ratio in both problems.

TheOREM 8 (Essentially [3]). There is an $\Omega(\log n / \log \log n)$ lower bound on the competitive ratios of DownWARD-ClOSED Prophet and Downward-Closed SECRETARY.
Proof SKeTCH. Let the feasible set system be a partition of the $n$ items into disjoint sets of size $\log n / \log \log n$. Let the value of each item be 1 with probability $\log \log n / \log n$, and 0 otherwise. With high probability, for at least one of the feasible sets, all items have value 1 ; thus the expected offline optimum is approximately $\log n / \log \log n$. However, after we select an item from any feasible set, the expected total value of the remaining items in this set is less than 1 . Thus no online algorithm can achieve expected value more than 2 . 